\title{
Community detection of political blogs network based on structure-attribute graph clustering model
}

\author{
Ahmed F. Al-Mukhtar, Eman S. Al-shamery \\ Department of Software Engineering, University of Babylon, Iraq
}

\begin{tabular}{l} 
Article Info \\
\hline Article history: \\
Received May 15, 2018 \\
Revised Nov 12, 2018 \\
Accepted Dec 11, 2018 \\
\hline Keywords: \\
Collaborative similarity \\
Community detection \\
Complex networks \\
Graph clustering \\
Social networks
\end{tabular}

\begin{abstract}
Complex networks provide means to represent different kinds of networks with multiple features. Most biological, sensor and social networks can be represented as a graph depending on the pattern of connections among their elements. The goal of the graph clustering is to divide a large graph into many clusters based on various similarity criteria's. Political blogs as standard social dataset network, in which it can be considered as blog-blog connection, where each node has political learning beside other attributes. The main objective of work is to introduce a graph clustering method in social network analysis. The proposed Structure-Attribute Similarity (SASCluster) able to detect structures of community, based on nodes similarities. The method combines topological structure with multiple characteristics of nodes, to earn the ultimate similarity. The proposed method is evaluated using well-known evaluation measures, Density, and Entropy. Finally, the presented method was compared with the state-of-art comparative method, and the results show that the proposed method is superior to the comparative method according to the evaluations measures.
\end{abstract}

Copyright $(0) 2019$ Institute of Advanced Engineering and Science. All rights reserved.

\section{Corresponding Author:}

Ahmed F. Al-Mukhtar,

Department of Software Engineering,

University of Babylon,

Hilla, Babylon, Iraq.

Email: ahmed.almukhtar@uokerbala.edu.iq

\section{INTRODUCTION}

In recent years, social networks are significantly considered as an important domain of complex networks, where networks can be modeled as graphs [1], [2]. The graph structure is a useful approach for studying social networks, where objects (such as people and authors) can be modeled as nodes and the relationships among objects can be represented as edges connecting these nodes. In social networks analysis, the graph clustering [3] is a great tool that is widely used to partition the large network into several densely connected community structures based on similarity measures. In result, the partitioned structures facilitate the understanding of large network visualization and make it easier to be analyzed.

The strategy of the graph clustering was used in many domains of social network analysis including, biological networks [4], community detection [5]-[9], and websites social networks [10]. Mainly, there are several graph clustering techniques, most of these techniques consider only the similarity of the topological structures [11], [12], others focus on the attributes of the of contents of the nodes [13], while few of them have considered both approaches [8].

Nowadays, and with increasing the influence of the weblogs on the human lifestyle especially in the periods of the US elections. Thus, it is the time to develop appropriate methods that are able to detect the community structures within these weblog networks. As a result, make it easier to visualize and analyze such networks. In this work, a new graph clustering method is proposed for community detection in social networks called Structure-Attribute Similarity Clustering (SAS-Cluster), that take into the account the 
similarities of the topological structures and the nodes attributes. Two concepts are introduced in this paper the Mean Gravity and the Path Degree, which are used to increase the community structure cohesiveness. The contributions of this paper are summarized below:

a. A new graph clustering algorithm is proposed which considers the structural similarity and the nodes attributes.

b. Two concepts (Mean Gravity and Path Degree) are introduced which are used to increase the cohesiveness of the structure of the clusters.

The remaining sections of this paper are arranged as follows. Section 2 introduces a review of some most recent related works. Section 3 focuses on the graph clustering technique. Section 4 describes Political Blogosphere Network. Section 5 introduces the proposed method. Section 6 reviews the experimental results. Finally, section 7 concludes this paper.

\section{RELATED WORKS}

This section reviews a summary of most recent related works concerning the graph clustering methods of social networks. The goal of graph clustering is to group the nodes of the network that have denser connections among them. Some methods such as Clique Peculation Methods CPM focus on internal/external edge counting [3] while ignoring the interactions and vertex characteristics, in [14] the authors propose a clique method on co-purchased network weighted graph, to find micro-cluster, the algorithm works in two phases graph polishing to enumerate intersections of neighbors and clique enumeration to count maximum cliques.

Newman-Girvan is considered as well-known divisive algorithm [15] for community detection which based on two main steps; first detects some edges based on betweenness measure then splits the network into communities based on the detected edges finally it requires betweenness recalculation after each splitting, the quality of the communities is measured using the maximal modularity. However, the method is not suitable for large networks and it suffers from the resolution limit.

In $\mathrm{ABCD}$ [6], the authors introduced new algorithm based on bi-directional connections and nodes features to detect community attractiveness of OSN, the algorithm was validated in SNAP platform and compared with CNM [5], according to the researcher ABCD is outperformed CNM and it can discover smaller communities in contrast with CNM, however, the method was not shown the comparative results of the modularity values to prove its effectiveness.

The k-prototype algorithm ISCD+ [16] an iterative model for fast graph clustering, the authors introduce a new idea for detecting communities, the algorithm imposes two factors namely local importance and importance concentration to select nodes with different weights to represent communities.

The KNN-based algorithms in [12] the authors propose a directed weighted graph clustering algorithm for community detection, the algorithm considers network topology only and it is significantly focused on the path traversed frequency and neighborhood nodes, nevertheless, the method suffers from computational complexity since it is based on k-nearest neighbors' computations.

In [10] the authors introduced a new approach for community detection in social network websites. besides structure similarity a frequent pattern mining of nodes contents was contributed, the algorithm is implemented in four steps, preprocessing, frequent pattern computing to obtain harmonious groups, extending harmonious groups into small communities, finally small communities expansion, however, the method suffers from some disadvantages such as, time complexity which is caused by the input parameters, a trial, and error concept was used to determine the appropriate parameters.

Roy et al. in [17] proposed a graph-based spectral clustering model, the method uses novel affinity matrix for spatial clustering with Mahalanobis distance, however, the method has some limitations, the distance metric can only measure from a single point, this reduces results quality.

Jinarat et al. in [18] have introduced a graph clustering algorithm for web search results, the core idea of the method is to combine web search results with external knowledge data from Wikipedia to attain better clustering quality. the method uses graph-based construction for text clustering to connect related documents, nevertheless, the similarity threshold parameter for subgraph detection must be in a certain range, when the threshold parameter increases, the clustering quality decreases.

\section{GRAPH CLUSTERING TECHNIQUE}

An indirect weighted graph $G=\{V, E, W, \Lambda\}$, where $|V|$ is a set of vertices. $|E|$ set of edges. $|W|$ set of edges weights. Each edge $e_{i} \in E \mid$ maps two vertices $\left(v_{i}, v_{j}\right)$ to be connected with specific weight $w_{i j}$, where $v_{i}, v_{j} \in V \mid$ and $w_{i j} \in W \mid$. Each vertex in a graph is associated with a set of attributes, in such that the 
term $\Lambda=\left\{a t t r_{1}, a t t r_{2}, a t t r_{3}, \cdots, a t t r_{n}\right\}$ where $\Lambda\left(v_{i}\right)=\bigcup_{j=1}^{n} v_{i} a t t r_{j}$ The purpose of graph clustering is to partition a graph into k-disjoint subgraphs, depending on some topological structures and attributes similarity measures, the communities should have the following aspects;

a. Similar vertices should be participated in a similar group, while the dissimilar ones should go to different groups.

b. The vertices that belong to the community should be densely connected to each other and sparsely connected to the other vertices within different communities.

The goal of the proposed algorithm is to introduce a weighted measure. Thus can effectively reflects the characteristics of network topology and vertices features to strengthen the similarity cohesiveness. The strategy of clustering and the similarity measure will be discussed in the next section.

\subsection{Contrast comparative method}

W-Cluster [9] is an emerged algorithm of SA-Cluster, which considers both structures and attributes aspects by applying a unified distance measure and neighborhood random walk strategy. The method uses the probability of edge belongs to the community to Measure link strength and Jaccard coefficient similarity to estimate content similarity. Eventually, W-cluster can automatically learn the degree of both topological similarities and attribute similarity through utilizing the probability transition matrix to build unified distance measure. The method partition large graph into numbers of clusters.

\subsection{Evaluation measures}

To evaluate the clusters quality results of the SAS-Cluster, two evaluation measures are used for this purpose; Density [1] and Entropy [19]. Both measures have the following definitions. Density measure is used to estimate the structural closeness to each cluster. Density is denoted in Equation (1).

$$
\operatorname{Density}\left(\left\{v_{c}\right\}_{c=1}^{k}\right)=\sum_{c=1}^{k} \frac{\left(v_{m}, v_{n}\right)}{|E|}
$$

where $\mathrm{c}$ is a number of clusters $\{1,2,3, \ldots, k\}, v_{c}$ represents the number of vertices belongs to the certain cluster. $v_{m}, v_{n}$ are two vertices, $v_{m}, v_{n} \in V \mid$ and $\left(v_{m}, v_{n}\right) \in v_{c} .\left(v_{m}, v_{n}\right)$ represents an edge $\in|E|$.

Entropy measure is used to determine the relevance of attributes among vertices in each cluster. Entropy can be defined in Equation (2) and Equation (3).

$$
\begin{aligned}
& \delta\left(\left\{V_{c}\right\}_{c=1}^{k}\right)=\sum_{c=1}^{m}\left(\frac{w_{a c}}{\sum_{l=1}^{m} w_{a l}} \sum_{i=1}^{k} \frac{\left|v_{i}\right|}{|V|} E\left(a c, v_{i}\right)\right) \\
& E=\left(a c, v_{i}\right)=-\sum_{n=1}^{n c} P_{c i n} \log _{2}\left(P_{c i n}\right)
\end{aligned}
$$

where $i$ is the number of clusters in the range $\{1,2,3, \ldots, k\}, a c$ is the attribute value at index number in range the $[1,2,3, \ldots, m] . n$ is the attribute values. $m, n c$ is a number of attribute values. $P_{c i n}$ is the percentage of cluster vertices that have $n^{\text {th }}$ attribute value on $a c$

\section{POLITICAL BLOGOSPHERE NETWORK}

The political web-blogs have played an important role in US Presidential Elections since the year of 2000 and after, and it is gained more influence at the 2004 US Presidential Elections. First, the blog can be understood as an informational website placed on World Wide Web WWW which is devoted to publishing a diary-style text or posts, sometimes contains links to other websites.

According to Adamic et al. in [20] the year 2004 demonstrated a rapid increase in the popularity of blogs, accordingly, the significant fraction of internet traffic was directed to these blogs. However, there is 
$9 \%$ of internet users acknowledged that they read political blogs during US Political Campion. Therefore, the weblogs may be followed by a small number of readers but its influence extends beyond that.

To discover the behavior of Blogosphere network, Adamic et al. in [20], have analyzed the landscape of most influential political blogs in two months before the US Presidential Elections. The analysis was based on the topics of the discussion and linking structure among blogs. The top 40 influential blogs were considered and added to the original list.

According to Adamic et al. a set of URLs was gathered from seven online weblog directories including, eTalkingHead, BlogCatalog, CampaignLine, and Blogarama. Each URL represents the political weblog. A one-day snapshot is taken for URLs, for each downloaded page, the citation was considered, and any newly discovered page was added to the list.

Next step, for all the discovered blogs, the citations are counted up, if the discovered page was cited for 17 times or more, then, its orientation is labeled manually depending on blogrolls and posts and added to the original list. The final set consists of 1494 blogs, divided into 759 liberals and 735 conservative blogs. The pattern in which the blogs are linked together was done, by counting the number of posts in such that each blog cites to another blog is counted as an edge between the two blogs. However, the link was not duplicated if the blog was cited by another blog more than once within the same post.

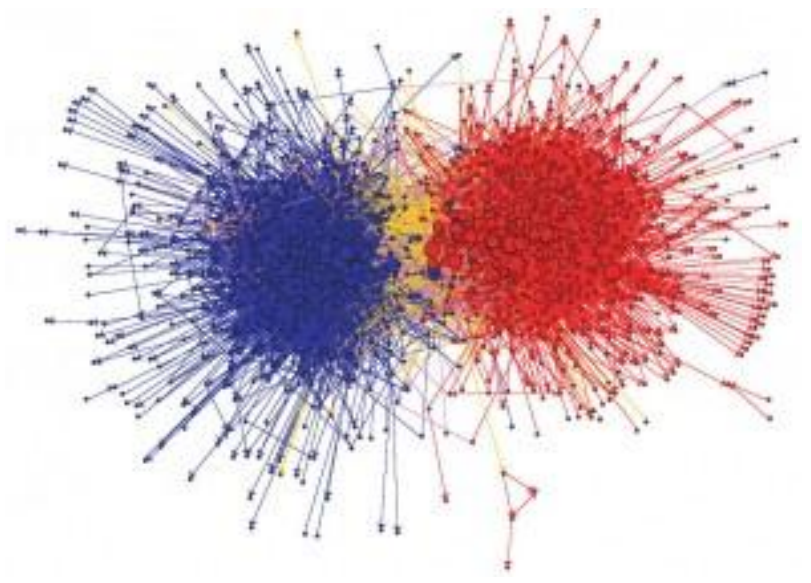

Figure 1. The community structures of the political blogs extracted from [20]. The colors reflect political orientations, the red for conservatives, the blue for liberals, the color purple from conservatives to liberals and the color orange from liberals to conservatives

Finally, the authors concluded network description, in such that, each political learning is more likely to talk about certain topics, one can notice an interesting pattern which is conservative bloggers tend to link to other conservative blogs and it is more densely linked (Table 1).

Table 1. Frequently used Items and their Descriptions

\begin{tabular}{cl}
\hline \multicolumn{1}{c}{ Symbols } & \multicolumn{1}{c}{ Description } \\
\hline$v_{m} \leftrightarrow v_{n}$ & Vertices with a direct connection \\
$v_{m} \rightarrow v_{n}$ & Vertices with an indirect connection \\
$v_{m} \phi v_{n}$ & Disconnected vertices \\
$d\left(v_{m}\right)$ & Number of ties connected to vertex $\mathrm{m}$ \\
$c\left(v_{m}\right)$ & Closeness centrality to vertex j \\
$S$ & Topological structure similarity \\
$A$ & attribute similarity \\
\hline
\end{tabular}




\section{THE PROPOSED METHOD}

\subsection{Block diagram}

In Figure 2 we can see the SAS-Cluster block diagram.

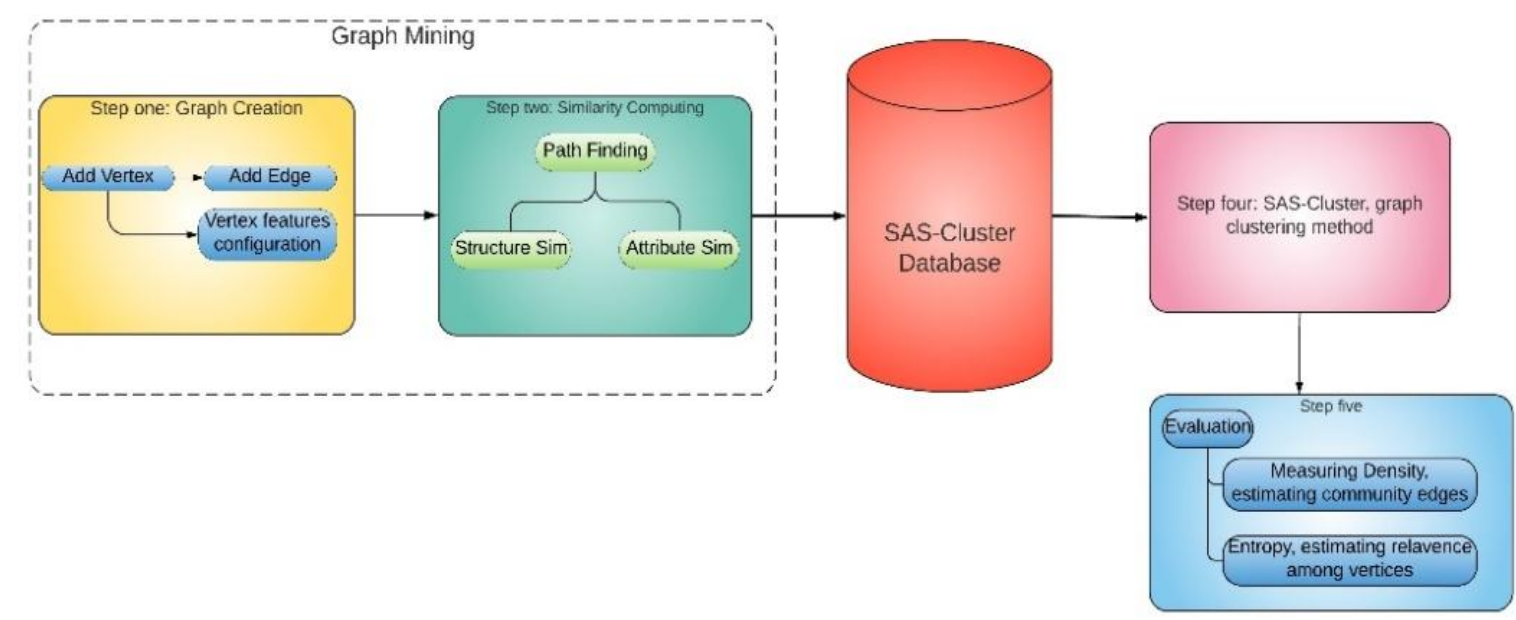

Figure 2. SAS-Cluster block diagram

\subsection{SAS-cluster}

Graphically, social networks can be modeled as complex networks, where both network topology and vertex properties can be contained. The relationship among vertices is represented as edges. The proposed SAS-Cluster algorithm achieves the following properties:

a. The vertices within the same cluster are close to each other concerning the structural similarity and dissimilar to other vertices outside the cluster.

b. vertices in the same cluster are close to each other in terms of attribute similarity and far from each other among the different clusters.

The core idea is to define the Gravity factor, to identify the power of the relationship concerning each pair of directly connected vertices in the topology of the structure.

Definition 1 (Gravity Factor). In the indirect weighted graph, the relation between two directly connected vertices exposes the strength of the relationship. Let $v_{m}$ and $v_{n}$ are two directly connected vertices. $d\left(v_{m}\right)$ is the degree of the vertex $v_{m} . c\left(v_{i}\right)$ is considered the closeness measure of $v_{i}$, which refers to the inverse sum of all shortest paths among $v_{i}$ and all other vertices in the graph. $w_{m n}$ is the weight associated with the edge $e\left(v_{m}, v_{n}\right)$. The Gravity Factor is defined in Equation (4).

$$
g\left(v_{m}, v_{n}\right)=\ln \left(1+\frac{d\left(v_{m}\right)}{\sum_{j=1}^{d\left(v_{m}\right)} c(j)} * w_{m n}\right), v_{m} \leftrightarrow v_{n}
$$

Definition 2 (Mean Gravity). Let $v_{m}$ and $\mathrm{v}_{\mathrm{n}}$ are two directly connected vertices. Mean gravity can be defined in Equation (5).

$$
m\left(v_{m}, v_{n}\right)=\frac{g\left(v_{m}, v_{n}\right)+g\left(v_{n}, v_{m}\right)}{2}, v_{m} \leftrightarrow v_{n}
$$

where $\mathrm{g}\left(\mathrm{v}_{\mathrm{n}}, \mathrm{v}_{\mathrm{m}}\right) \neq \mathrm{g}\left(\mathrm{v}_{\mathrm{m}}, \mathrm{v}_{\mathrm{n}}\right)$, thus one can determine which vertex is more important.

Definition 3 (Path Degree). Let $v_{m}$ and $\mathrm{v}_{\mathrm{n}}$ are two indirectly connected vertices. For a given path $\left(v_{m}, v_{m+1}, v_{m+2}, \ldots, v_{m+i}, v_{n}\right)$, path degree can be defined in Equation (6). 


$$
p\left(v_{m}, v_{n}\right)=\prod_{v_{m}}^{v_{n}} g\left(v_{m}, v_{n}\right), v_{m} \leftrightarrow v_{n}
$$

where path is taken into account as the weighted shortest path between a pair of indirectly connected vertices. Structural/Attribute Similarity (SAS), in the proposed method, the Jaccard similarity coefficient is adopted to compute the similarity measure, as defined in Equation (7).

$$
\operatorname{sim}(X, Y)=\frac{|X \bigcup Y|}{X \cap Y}
$$

where $X, Y$ are vertices, $X, Y \in|V|$, Jaccard similarity in the equation (7) is a well-known similarity measure, therefore it has been used to find out the relevance among vertices. There are two main similarity calculations are taken into account. Directly connection Equation (8). To calculate the similarity between a pair of directly connected vertices.

$$
\operatorname{sim}\left(v_{m}, v_{n}\right)=\frac{w_{m n}}{\sum_{i=1}^{v_{m}} w_{m i}+\sum_{j=1}^{v_{n}} w_{n j}-w_{m n}}, v_{m} \leftrightarrow v_{n}
$$

where $v_{m}, v_{n} \in|V| . w_{m i}$ is the weight of the edge between the vertex $v_{m}$ and all $i^{S}$ vertices that are directly connected to $v_{m} . w_{n j}$ is the weight of the edge between the vertex $v_{n}$ and all $j^{s}$ vertices that are directly connected to $v_{n}$ and $w_{m n}$ is the associated weight of the edge $e\left(v_{m}, v_{n}\right)$. Indirectly connection Equation (9). The similarity is calculated based on the shortest path between $v_{m}$ and $v_{n}$.

$$
\operatorname{sim}\left(v_{m}, v_{n}\right)=\prod_{l=v_{m}}^{v_{n}} \operatorname{sim}\left(v_{l}, v_{l+1}\right), v_{m} \rightarrow v_{n}
$$

where $v_{m}, v_{n} \in|V|$ represent the similarity between two indirectly connected vertices.

After obtaining the Mean Gravity Equation (5) and Path Degree Equation (6), the structural similarity is defined in Equation (10).

$$
\operatorname{sim}\left(v_{m}, v_{n}\right)_{S}=\left\{\begin{array}{c}
\operatorname{sim}\left(v_{m}, v_{n}\right)+m\left(v_{m}, v_{n}\right), v_{m} \leftrightarrow v_{n} \\
\operatorname{sim}\left(v_{m}, v_{n}\right)+p\left(v_{m}, v_{n}\right), v_{m} \rightarrow v_{n} \\
0, \quad v_{m} \phi v_{n}
\end{array}\right.
$$

Next, the vertices attributes are considered. Each vertex is characterized by multiple attributes. Obtaining attribute similarity increases the cohesiveness among vertices. Attributes values can be either 1 or 0 reflect the appearance or disappearance of that attribute at a certain vertex. The mathematical formulations of the attribute similarity are defined in Equation (11) and Equation (12).

$$
\vartheta\left(v_{m}, v_{n}\right)=\left\{\begin{array}{lr}
1, \text { if } v_{m}, v_{n} \text { have value on } i^{\text {th }} \text { attr } \\
0, & \text { otherwise }
\end{array}\right.
$$




$$
\operatorname{sim}\left(v_{m}, v_{n}\right)_{A}=\left\{\begin{array}{c}
\frac{\sum_{i=1}^{|m|} \vartheta\left(v_{n}, v_{m}\right) \times w_{a i}}{\sum_{j=1}^{|m|} w_{a j}}, v_{m} \leftrightarrow v_{n} \\
\prod_{l=v_{n}}^{v_{n}} \operatorname{sim}\left(v_{l}, v_{l+1}\right)_{A}, v_{m} \rightarrow v_{n} \text { and } v_{l} \in V
\end{array}\right.
$$

where $v_{m}, v_{n} \in|V| . m$ is the number of attributes associated with $v_{m} . w_{a}$ represents the attribute value usually either 0 or 1 . By combining Equation (10) and Equation (12) the final mathematical description of the structure attribute similarity is defined in Equation (13).

$$
\operatorname{SAS}\left(v_{m}, v_{n}\right)=\alpha \times \operatorname{sim}\left(v_{m}, v_{n}\right)_{s}+\beta \times \operatorname{sim}\left(v_{m}, v_{n}\right)_{A}
$$

where $S A S\left(v_{m}, v_{n}\right)$ is the structure attribute similarity between a pair of vertices $v_{m}$ and $v_{n}$.

\subsection{Algorithm description}

After obtaining final similarity value among vertices, by applying Equation (13). now one can get the distance value among vertices which is defined in Equation (14), but first, the similarity value has to be normalized to be in the range of $[0,1]$.

$$
S A S-D\left(v_{m}, v_{n}\right)=\left\{\begin{array}{l}
1-\operatorname{norm}(S A S) \\
\infty, \quad v_{m} \phi v_{n}
\end{array}\right.
$$

$S A S-D\left(v_{m}, v_{n}\right)$ is the Distance value between the two vertices $v_{m}$ and $v_{n} . d\left(v_{m}\right) \neq 0$ and $d\left(v_{n}\right) \neq 0$.

In prior, the number of centroids $\mathrm{k}$ are selected randomly, at each iteration these centroids are updated, the rest of vertices are assigned to the nearest centroid based on minimum distance.

\section{Algorithm 1. SAS-Cluster}

Input: undirected, weighted* and multi-attribute Graph $G$, clusters number $k$, the weight factor $\alpha$, max iteration number $M A X$

Output: $k$-clusters $C_{1}, C_{2}, \cdots C_{k}$

1. Initialization: vertices $\left\{v_{i}\right\}_{i=1}^{n}=0$, distance $D\left[v_{i}\right]\left[v_{j}\right]=0$, vertices attributes $\left[v_{i, \text { wattrj }}\right]_{j=1}^{m}=0$, $\beta=1-\alpha$, ClusterSentroid []$=0$.

2. Graph creation:

2.1. Add vertex:

$$
\begin{aligned}
& \text { If NewNode not in vertices }\left\{v_{i}\right\} \\
& \text { Vertices }\left\{v_{i}\right\} \leftarrow \text { NewNode } \\
& \text { End if } \\
& \text { 2.2. Add Edge } \\
& \text { If } v_{i}, v_{j} \text { in vertices }\left\{v_{i}\right\} \\
& \text { Create }\left(v_{i}, v_{j}\right) \text { link \& }\left(v_{j}, v_{i}\right) \text { link } \\
& \text { End if }
\end{aligned}
$$

3. Similarity computation:

For each vertex $v_{i} \& v_{j}$ in $|V|$ where $i \neq j$

$$
\begin{aligned}
& S A S\left(v_{i}, v_{j}\right)=\alpha \times \operatorname{sim}\left(v_{i}, v_{j}\right)_{s}+\beta \times \operatorname{sim}\left(v_{i}, v_{j}\right)_{A} \\
& S A S-D\left(v_{i}, v_{j}\right)=1-\operatorname{Norm}\left(\operatorname{SAS}\left(v_{i}, v_{j}\right)\right)
\end{aligned}
$$

End for
4. SAS-Cluster:

$$
\text { Select } k \text { randomly from vertices }|V| \text { as initial centroids for } C_{1}, C_{2}, \cdots C_{k}
$$

ClusterSentroid []$=k\left(c_{k}\right)$

While not end OR there is no change in the MIN distance 


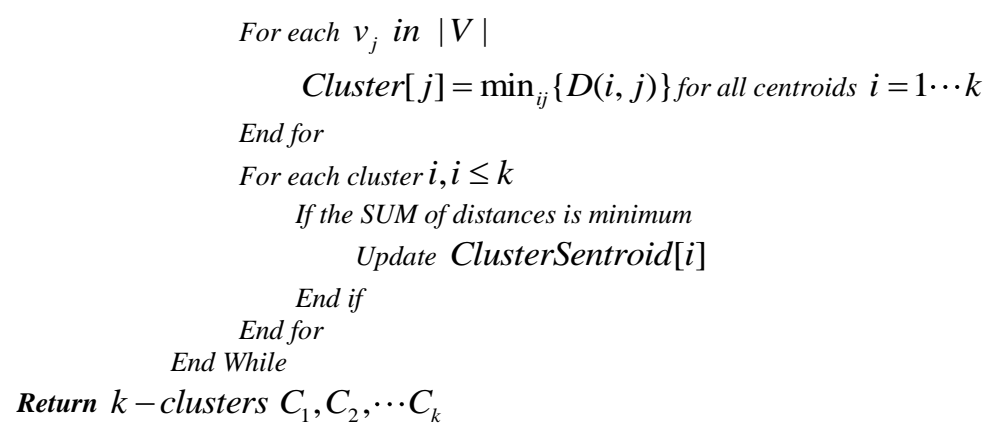

Initially and regarding

Figure 2, SAS-Cluster algorithm requires two predetermined parameters $\alpha$ and number of clusters $k$. at step one algorithm reads the raw data to create the network and determines the number of attributes associated with each vertex. Step two multiple paths among nodes are considered, many calculations must be insured, Mean Gravity Equation (5) and Path Degree Equation (6), are required to establish SAS similarity calculations as in Equation (13), Distance values among each couple of vertices in Equation (14) are computed based on SAS similarity results. All data must be stored in the database. Step four and regarding the number of $k$ and distance values, the communities are extracted from the original graph. Finally, and concerning the Equations (1), (2) and (3), the results are evaluated using Density and Entropy.

\section{EXPERIMENTAL RESULTS}

In this section, extensive experiments are performed to evaluate the performance of the SAS-Cluster method. All experiments are conducted on PC with Windows 10 Pro 64 bit, an i7-6700 HG CPU (260 GHz, and 16 GB RAM. The programming environment is Python 3.6.2 (MSC v.1900 32 bit (Intel)).

\subsection{Dataset}

Political Blogs Dataset, as real network dataset, which is used to for the evaluation and analyzing the proposed method. The dataset is based on blog-blog connection [20]. It consists of 1,490 nodes and each node contains an attributes description to characterize its political learning, which is either conservative or democrat.

\subsection{Results}

The proposed SAS-Cluster algorithm is extensively evaluated with the state-of-art method W-cluster [9] through well-known evaluating measures, Density, and Entropy. The density as given in Equation (1), reflects the extent of how tight structure is connected among vertices in each cluster, the higher density value reflects the community structure cohesiveness. The entropy that is described in Equation (2) and Equation (3), which is used to rate the attribute relationship among vertices, low entropy reflects better relevance among vertices in each cluster. Figure 3 and Figure 4 show the performance of SAS-Cluster concerning Density and Entropy, where the number of clusters $k=3,5,7,9 . \alpha$ is set in the range $[0,1]$ and $\beta=1-\alpha$. The algorithm is run for at least three iterations.

Figure 3, reviews the density values. When setting $\alpha$ to 0 the density value is the lowest, this because of the similarity of the structural topology is not taken into account. At $k=3$ the density values declines when $\alpha$ is set to 0.6 or 0.7 . At $k=5,7,9$ and $\alpha<0.5$ the density values drops down.

Figure 4 , reviews the entropy values, the best-given values when $\alpha$ equal to 0 , since the algorithm is run based on the attribute similarity only. In contrast, when $\alpha$ equal to 1 the given-values is the worst this because the attribute similarity is not taken into the account. At $k=3,5,7,9$ the best given-results when $\alpha$ is set to 0.5 or 0.8 . While the quality of the results tends to decrease when $\alpha>0.8$. As illustrated in Figure 3 and Figure 4, the best performance for SAS-Cluster when $\alpha$ is either 0.5 or 0.8 . 


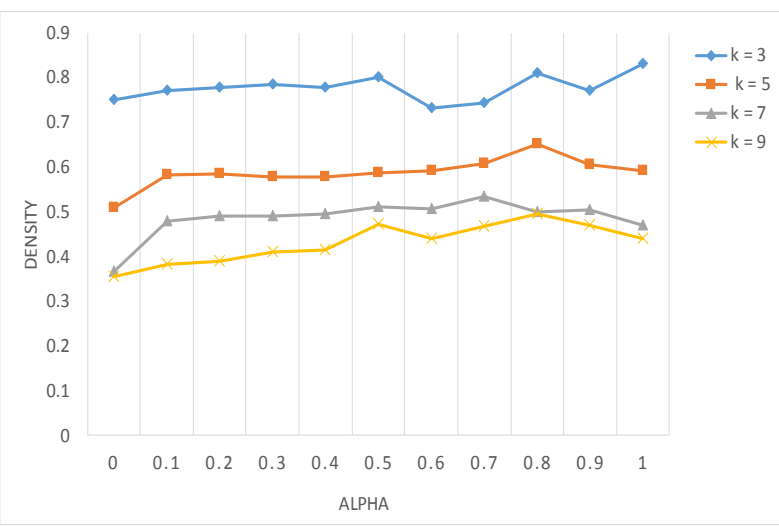

Figure 3. Impact factor $\alpha$ on the political blog. Clarifies Density results for SAS-Cluster

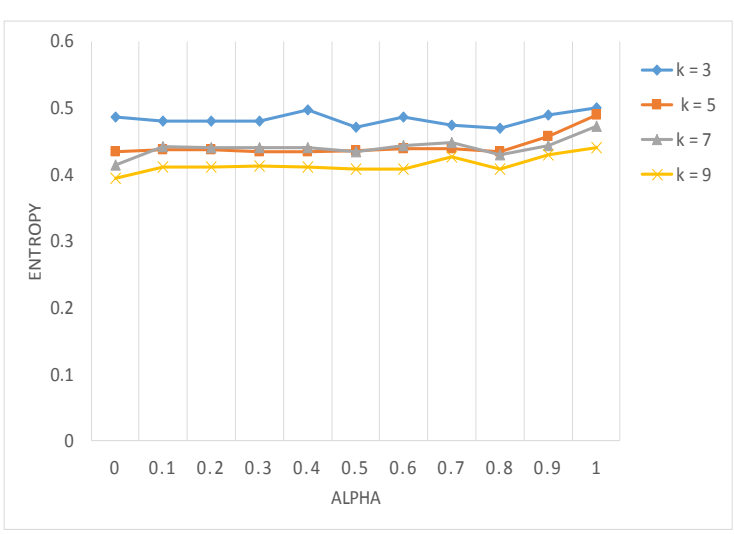

Figure 4. Impact factor $\alpha$ on the political blog. Clarifies Entropy results for SAS-Cluster

To show the effectiveness of the proposed method, SAS-Cluster is compared with the state-of-art method, W-cluster. Both methods are tested for a fixed number of clusters $k=3,5,7,9$ and $\alpha$ is set to 0.5 .

Figure 5 and Figure 6 illustrate the comparison results of the density and the entropy respectively for each of SAS-Cluster and W-cluster. All results have shown that SAS-Cluster outperformed W-Cluster concerning the density and the entropy measures.

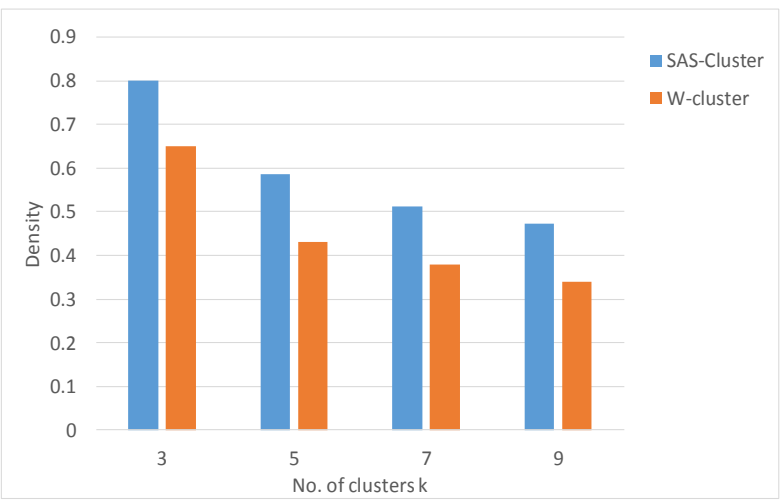

Figure 5. Density comparison values on political blogs

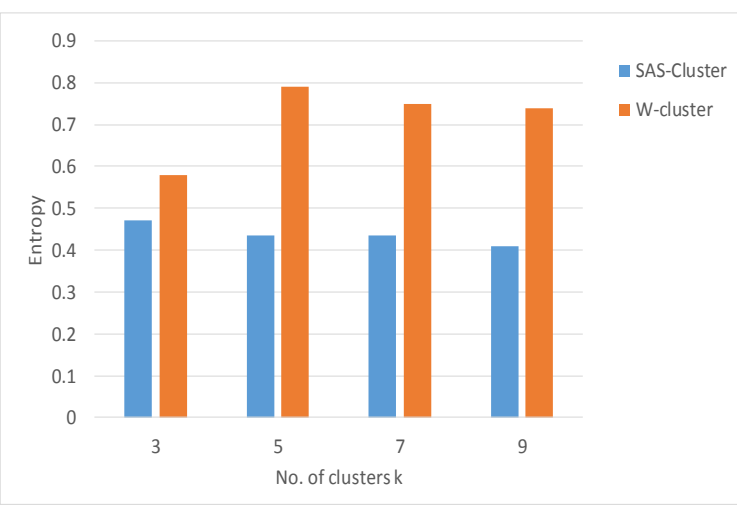

Figure 6. Entropy comparison values on political blogs

\section{CONCLUSION}

Nowadays, social networks have become more influential in individual's opinion, decisions, and their lifestyle. Therefore, and with the accelerated increase in social networks data, it is important to adopt a more reliable graph clustering methods for community detection. In this paper, a graph clustering method for community detection is proposed. The method introduces two concepts, Gravity degree and Path degree, to increase the structural similarities within the detected communities. In addition, the adopted method combines structural similarities with the multiple attributes of nodes to attain more cohesiveness similarity. The experimental results have shown that SAS-Cluster is better than W-cluster according to Density and Entropy evaluation measures.

\section{REFERENCES}

[1] P. Mrutyunjaya, et al., "Social Networking," Book of Springer International Publishing, vol. 65, pp. 45-83, 2014.

[2] S. S. Elisa, "Graph Clustering," Computer Science Review, vol. 1, pp. 27-64, 2007.

[3] F. Santo and H. Darko, "Community detection in networks: A user guide," Physics Reports, vol. 659, pp. 1-45, 2016.

[4] Girvan M. and Newman M. E., "Community structure in social and biological networks," Proceedings of the national academy of sciences, vol. 99, pp. 7821-7826, 2002.

[5] Clauset, et al., "Finding community structure in very large networks," Physical review E, vol. 70, pp. 1-6, 2004.

Community detection of political blogs network based on structure-attribute... (Ahmed F. Al-Mukhtar) 
[6] Liu, et al., "Weighted Graph Clustering for Community Detection of Large Social Networks," Procedia Computer Science, vol. 31, pp. 85-94, 2014.

[7] Lambiotte, et al., "Random Walks, Markov Processes and the Multiscale Modular Organization of Complex Networks," IEEE Transactions on Network Science and Engineering, vol. 1, pp. 76-90, 2014.

[8] Boobalan, et al., "Graph clustering using k-Neighbourhood Attribute Structural similarity," Applied Soft Computing, vol. 47, pp. 216-223, 2016.

[9] Cheng, et al., "Clustering Large Attributed Graphs: A Balance between Structural and Attribute Similarities," ACM Transactions on Knowledge Discovery from Data, vol. 5, pp. 1-33, 2011.

[10] Moosavi, et al., "Community detection in social networks using user frequent pattern mining," Knowledge and Information Systems, vol. 51, pp. 159-186, 2017.

[11] Chen, et al., "Study on similarity based on connection degree in social network," Cluster Computing, vol. 20, pp. 167-178, 2017.

[12] Parimala, et al., "K-Neighbourhood Structural Similarity Approach for Spatial Clustering," Indian Journal of Science and Technology, vol. 8, 2015.

[13] Tian, et al., "Efficient aggregation for graph summarization," Proceedings of the 2008 ACM SIGMOD international conference on Management of data - SIGMOD '08, pp. 567-580, 2008.

[14] Yamazaki, et al., "Weighted Micro-Clustering: Application to Community Detection in Large-Scale Co-Purchasing Networks with User Attributes," Proceedings of the 25th International Conference Companion on World Wide Web, pp. 131-132, 2016.

[15] Newman M. E. and Girvan M., "Finding and evaluating community structure in networks," Physical Review E, vol. 69, pp. 026113, 2004.

[16] Bai, et al., "Fast graph clustering with a new description model for community detection," Information Sciences, vol. 388-389, pp. 37-47, 2017.

[17] Mandal J. K. and Roy P., "A novel spectral clustering based on local distribution," International Journal of Electrical and Computer Engineering, vol. 5, pp. 361, 2015.

[18] J. Supakpong and H. Choochart, "Graph-Based Concept Clustering for Web Search Results," International Journal of Electrical and Computer Engineering, vol. 5, pp. 1536-1544, 2015.

[19] F. Santo, "Community detection in graphs," Physics Reports, vol. 486, pp. 75-174, 2010.

[20] Adamic, et al., "The political blogosphere and the 2004 US election: divided they blog," Proceedings of the 3rd international workshop on Link discovery, pp. 36-43, 2005. 